\title{
A flexible ranked set sampling scheme: Statistical analysis on scale parameter
}

\author{
Abbas Eftekharian, Mostafa Razmkhah*, and Jafar Ahmadi \\ Department of Statistics, Ferdowsi University of Mashhad, Iran
}

\begin{abstract}
A flexible ranked set sampling scheme including some various existing sampling methods is proposed. This scheme may be used to minimize the error of ranking and the cost of sampling. Based on the data obtained from this scheme, the maximum likelihood estimation as well as the Fisher information are studied for the scale family of distributions. The existence and uniqueness of the maximum likelihood estimator of the scale parameter of the exponential and normal distributions are investigated. Moreover, the optimal scheme is derived via simulation and numerical computations.
\end{abstract}

Keywords Fisher information; Maximum likelihood estimation; Optimality; Order statistics; Relative efficiency.

AMS 2010 subject classifications 62D05, 62F10

DOI: $10.19139 /$ soic-2310-5070-812

\section{Introduction}

Ranked set sampling (RSS) was introduced by [14] as a method for unbiased selective sampling. In this scheme, $k$ independent sets each contains $k$ units are randomly selected from the population of interest. Next, the units of each set are visually sorted in ascending order and only the $j$ th $(j=1,2, \ldots, k)$ judgment order statistic of the $j$ th set is measured. When judgment ranking is accurate, we say the ranking is perfect and the selected units are actually a sample of $k$ independent order statistics. This scheme may be repeated at some cycles to attain a reasonable data set. [23] proved that the mean of RSS is an unbiased estimator of the population mean and its variance is always smaller than that of simple random sampling (SRS). This method can reduce cost of sampling and increase the accuracy of the results.

The same results were obtained by [12] by considering errors in judgment ranking. Some results of parametric RSS were presented by [22], [15], and [8]. The RSS scheme has been developed into various procedures. In this paper, we consider three existing types of ranking sampling schemes which are briefly explained as follows:

- Extreme ranked set sampling (ERSS): This procedure was introduced by [19] in estimating problem of the population mean which is similar to RSS but only minimum or maximum of each set is measured and it is assumed that they can be detected visually. Therefore, the judgment ranking error in ERSS is less than that of RSS. The procedure of obtaining such a sample depends on whether $k$ is even or odd. When $k$ is even, from the first $k / 2$ sets the lowest ranked units and from the last $k / 2$ sets the largest ranked units are measured. When $k$ is odd, the minimum of the first $[k / 2]$ sets and also the maximum of the last $[k / 2]$ sets are selected, where $[a]$ is the integer part of $a$, moreover, the median of the $((k+1) / 2)$ th set is measured.

\footnotetext{
*Correspondence to: Mostafa Razmkhah (Email: razmkhah_m@um.ac.ir), Department of Statistics, Ferdowsi University of Mashhad, P. O. Box 1159, Mashhad 91775, Iran.
}

ISSN 2310-5070 (online) ISSN 2311-004X (print)

Copyright (C) 2021 International Academic Press 
- Moving extreme ranked set sampling (MERSS): [2] introduced this scheme which is in fact an unbalanced ERSS. In this procedure, first of all, $k$ independent random sets are considered with sizes $1,2, \ldots, k$, sequentially. Then, the maximum of each set is determined by judgment, i.e., by visual inspection or by some other relatively inexpensive methods, without actual measurement of the variable of interest. Finally, the judgment maximum of each set is measured accurately. This procedure is called "MERSS type I". If instead of maximum in the mentioned procedure, the minimum of each set is measured, an "MERSS type II" scheme is obtained. Note that both of MERSS type I and MERSS type II may be performed to obtain an ordinary MERSS data set. This procedure seems suitable for the case of large set size, because it reduces the error of ranking and keeps optimality inherited in the ordinary RSS procedure. Therefore, in comparison with RSS, the MERSS is a useful modification in this case. [1] obtained maximum likelihood estimator (MLE), modified MLE and likelihood ratio test for scale parameter of exponential distribution using MERSS. The MLE and Modified MLE of scale parameter and their properties are studied by $[9,10]$.

- Median ranked set sampling (MRSS): This procedure was suggested by [16] for estimating the population mean. In MRSS scheme, $k$ sets of the same size $k$ are taken from the population of interest and the units in each set are ranked, visually. If $k$ is odd, the median of each set is measured. When $k$ is even, from the first $k / 2$ sets, the $(k / 2)$ th smallest ranked unit and from the last $k / 2$ sets the $((k+2) / 2)$ th smallest ranked unit are selected by judgment and measured accurately. [17] presented a test of hypothesis for the mean of the symmetric distributions based on MRSS.

Recently, [4] introduced truncation-based ranked set samples. They compared performance of mean and median of this scheme with those of SRS, RSS, ERSS and MRSS. Simultaneous comparison of the location and scale estimators based on SRS and RSS in two-parameter exponential distribution is performed by [20]. So far, there are several ranked set sampling schemes, but in some practice situations some plans may not be applicable at any cycle of the test. With this in mind, in this paper, a flexible RSS (FRSS) with $M$ cycles is considered which contains $m_{1}$ cycles of an usual RSS, the ERSS with $m_{2}$ cycles, the MERSS type I with $m_{3}$ cycles, the MERSS type II with $m_{4}$ cycles and the MRSS with $m_{5}$ cycles, such that $M=\sum_{i=1}^{5} m_{i}$. The proposed scheme is indeed a combination of several RSS schemes. For simplicity, throughout the paper, we assume that the set size $k$ is even for all cases. The proposed sampling scheme may be used to minimize the error of ranking and cost of sampling. Since in the ERSS, MERSS type I and MERSS type II, only the extreme order statistics (maximum or minimum) in each set are measured, the error of ranking is less than other schemes. So, if simultaneous use of these schemes leads to rather high efficiency, it may be suggested to combine some existing schemes in practice. Some special cases of FRSS lead to various types of existing sampling schemes; for example, if $m_{2}=m_{3}=m_{4}=m_{5}=0$, the ordinary RSS with $M=m_{1}$ cycles is derived. Nevertheless, combining some kinds of these schemes may lead to an optimal sampling scheme which is easier or cheaper to perform. It may also reduce the error of rankings. The proposed scheme has been previously introduced by [13] for estimating the population mean. They showed that combining some various existing schemes may lead to a more efficient estimator.

The rest of paper is organized as follows. In Section 2, some preliminaries are presented and the likelihood function of the FRSS scheme is introduced. Section 3 focuses on obtaining the MLE of scale parameter based on FRSS scheme. Also, existence and uniqueness of the MLE for the scale parameter of exponential and normal distributions are studied. The Fisher information (FI) contained in a FRSS is investigated in Section 4. In Section 5, the optimal FRSS scheme is determined. Toward this end, considering some different costs in addition to mean squared error (MSE) of MLEs, the relative efficiency (RE) of the FRSS scheme with respect to the SRS method is defined. In Section 6, some conclusions are stated.

\section{Preliminaries}

In this section, we briefly present some notations and axillary results. First of all, let us denote the cumulative distribution function (cdf) and probability density function (pdf) of the population by $F(x ; \boldsymbol{\theta})$ and $f(x ; \boldsymbol{\theta})$, 
respectively, where $\boldsymbol{\theta}$ is a vector of parameters. In the following, the data sets and likelihood functions concerning to different sampling schemes with $m$ cycles have been presented.

- Let us denote the data set of RSS by

$$
\mathbf{X}_{R S S}^{(m)}=\left\{X_{1(1: k) 1}, X_{2(2: k) 1}, \ldots, X_{k(k: k) 1}, \ldots, X_{1(1: k) m}, X_{2(2: k) m}, \ldots, X_{k(k: k) m}\right\},
$$

where $X_{j(j: k) i}$ is the $j$ th order statistic in a random sample of size $k$ at the $j$ th set of the $i$ th cycle for $i=1, \ldots, m$ and $j=1, \ldots, k$. The corresponding likelihood function is given by

$$
\begin{aligned}
L_{R S S}^{(m)}(\boldsymbol{\theta}) & =\prod_{i=1}^{m} \prod_{j=1}^{k} j\left(\begin{array}{c}
k \\
j
\end{array}\right) f\left(x_{j(j: k) i} ; \boldsymbol{\theta}\right)\left[F\left(x_{j(j: k) i} ; \boldsymbol{\theta}\right)\right]^{j-1}\left[\bar{F}\left(x_{j(j: k) i} ; \boldsymbol{\theta}\right)\right]^{k-j} \\
& =\left\{k ! \prod_{j=1}^{k}\left(\begin{array}{c}
k \\
j
\end{array}\right)\right\}^{m} \prod_{i=1}^{m} \prod_{j=1}^{k} f\left(x_{j(j: k) i} ; \boldsymbol{\theta}\right)\left[F\left(x_{j(j: k) i} ; \boldsymbol{\theta}\right)\right]^{j-1}\left[\bar{F}\left(x_{j(j: k) i} ; \boldsymbol{\theta}\right)\right]^{k-j},
\end{aligned}
$$

where $\bar{F}(\cdot)=1-F(\cdot)$ and $x_{j(j: k) i}$ is the observed value of $X_{j(j: k) i}$. For more details, see for example, [22] and [6]. Throughout this paper, the product $\prod_{i=1}^{0}$ is defined to be 1 .

- When the set size $k$ is even, assuming the data set of an ERSS is

$$
\begin{aligned}
\mathbf{X}_{E R S S}^{(m)}= & \left\{X_{1(1: k) 1}, X_{2(1: k) 1}, \ldots, X_{\frac{k}{2}(1: k) 1}, X_{\frac{k+2}{2}(k: k) 1}, \ldots, X_{k(k: k) 1}, \ldots,\right. \\
& \left.X_{1(1: k) m}, X_{2(1: k) m}, \ldots, X_{\frac{k}{2}(1: k) m}, X_{\frac{k+2}{2}(k: k) m}, \ldots, X_{k(k: k) m}\right\},
\end{aligned}
$$

the appropriate likelihood function is given by (see, 3 )

$$
L_{E R S S}^{(m)}(\boldsymbol{\theta})=\prod_{i=1}^{m}\left\{\prod_{j=1}^{\frac{k}{2}} k f\left(x_{j(1: k) i} ; \boldsymbol{\theta}\right)\left[\bar{F}\left(x_{j(1: k) i} ; \boldsymbol{\theta}\right)\right]^{k-1} \prod_{j=\frac{k}{2}+1}^{k} k f\left(x_{j(k: k) i} ; \boldsymbol{\theta}\right)\left[F\left(x_{j(k: k) i} ; \boldsymbol{\theta}\right)\right]^{k-1}\right\} .
$$

- Denoting the data sets of the MERSS type I and the MERSS type II by

$$
\mathbf{X}_{M E R S S-I}^{(m)}=\left\{X_{1(1: 1) 1}, \ldots, X_{k(k: k) 1}, \ldots, X_{1(1: 1) m}, \ldots, X_{k(k: k) m}\right\},
$$

and

$$
\mathbf{X}_{M E R S S-I I}^{(m)}=\left\{X_{1(1: 1) 1}, \ldots, X_{k(1: k) 1}, X_{1(1: 1) m}, \ldots, X_{k(1: k) m}\right\},
$$

then, the corresponding likelihood functions are

$$
L_{M E R S S-I}^{(m)}(\boldsymbol{\theta})=\prod_{i=1}^{m} \prod_{j=1}^{k} j f\left(x_{j(j: j) i} ; \boldsymbol{\theta}\right)\left[F\left(x_{j(j: j) i} ; \boldsymbol{\theta}\right)\right]^{j-1}
$$

and

$$
L_{M E R S S-I I}^{(m)}(\boldsymbol{\theta})=\prod_{i=1}^{m} \prod_{j=1}^{k} j f\left(x_{j(1: j) i} ; \boldsymbol{\theta}\right)\left[\bar{F}\left(x_{j(1: j) i} ; \boldsymbol{\theta}\right)\right]^{j-1},
$$

respectively. See, [1] and [9].

- Moreover, if the data set of MRSS is denoted by

$$
\mathbf{X}_{M R S S}^{(m)}=\left\{\left\{X_{j(k / 2: k) i}\right\}_{j=1}^{k / 2} \cup\left\{X_{j(k / 2+1: k) i}\right\}_{j=k / 2+1}^{k}, \quad i=1,2, \ldots, m\right\},
$$


where $A \cup B$ stands for the union of $A$ and $B$, the likelihood function is given by (see, 21)

$$
L_{M R S S}^{(m)}(\boldsymbol{\theta})=\prod_{i=1}^{m}\left\{\left[\prod_{j=1}^{\frac{k}{2}} f_{\left(\frac{k}{2}\right)}\left(x_{j\left(\frac{k}{2}: k\right) i} ; \boldsymbol{\theta}\right)\right]\left[\prod_{j=\frac{k}{2}+1}^{k} f_{\left(\frac{k}{2}+1\right)}\left(x_{j\left(\frac{k}{2}+1: k\right) i} ; \boldsymbol{\theta}\right)\right]\right\}
$$

where $f_{(j)}(\cdot)$ is the pdf of the $j$ th order statistic in a random sample of size $k$ from the pdf $f(\cdot)$.

As previously mentioned, a FRSS data set is obtained, when all the above mentioned sampling schemes are performed together with different cycles. Therefore, the associated data set is as follows

$$
\mathbf{X}_{F R S S}^{(M)}=\left\{\mathbf{X}_{R S S}^{\left(m_{1}\right)} \cup \mathbf{X}_{E R S S}^{\left(m_{2}\right)} \cup \mathbf{X}_{M E R S S-I}^{\left(m_{3}\right)} \cup \mathbf{X}_{M E R S S-I I}^{\left(m_{4}\right)} \cup \mathbf{X}_{M R S S}^{\left(m_{5}\right)}\right\}
$$

The sample size of a FRSS is equal to $M k$, where $M=\sum_{i=1}^{5} m_{i}$. In practice, we can select a permutation of $\left(m_{1}, m_{2}, m_{3}, m_{4}, m_{5}\right)$, that reduces the ranking error or cost of sampling. Here, $\left(m_{1}, \ldots, m_{5}\right)$ is called the FRSS scheme and its optimality is studied in comparison with SRS method in view of maximum RE by considering costs of sampling. Since the various data sets are independently obtained, the likelihood function of the FRSS scheme is given by

$$
L_{F R S S}^{(M)}(\boldsymbol{\theta})=L_{R S S}^{\left(m_{1}\right)}(\boldsymbol{\theta}) L_{E R S S}^{\left(m_{2}\right)}(\boldsymbol{\theta}) L_{M E R S S-I}^{\left(m_{3}\right)}(\boldsymbol{\theta}) L_{M E R S S-I I}^{\left(m_{4}\right)}(\boldsymbol{\theta}) L_{M R S S}^{\left(m_{5}\right)}(\boldsymbol{\theta}) .
$$

The MLE and FI under FRSS scheme are studied in the next sections.

\section{MLE of scale family based on FRSS}

We recall that for given pdf $f(x ; \sigma), \sigma$ is a scale parameter, when $f(x ; \sigma)=\frac{1}{\sigma} f_{1}\left(\frac{x}{\sigma}\right)$ where $\sigma>0$ and $f_{1}(\cdot)$ is a pdf and does not depend on $\sigma$. Then, $f(x ; \sigma)$ is called a scale family. For more details, see for example, Rohatgi and Ehsans Saleh (2015, p.196). There are many papers on the scale family as well as location or location-scale families of distributions based on various procedures of RSS scheme. The research work by [22] was one of the primary papers on estimation of location and scale parameters of distributions using RSS. [21] compared the MLE of location and scale parameters based on RSS, MRSS and ERSS. The MLE of scale parameter and its properties was studied by [9] on the basis of the MERSS scheme. Here, we investigate existence and uniqueness of MLE in a scale family based on FRSS procedure as proposed in previous section. In this case, using (1), the log-likelihood function of $\sigma$ is given by

$$
\begin{aligned}
l(\sigma)= & \log c-M k \log \sigma+\sum_{i=1}^{m_{1}} \sum_{j=1}^{k}\left\{\varphi_{1}\left(\frac{x_{j(j: k) i}}{\sigma}\right)+(j-1) \varphi_{2}\left(\frac{x_{j(j: k) i}}{\sigma}\right)+(k-j) \varphi_{3}\left(\frac{x_{j(j: k) i}}{\sigma}\right)\right\} \\
& +\sum_{i=1}^{m_{2}}\left\{\sum_{j=1}^{\frac{k}{2}}\left(\varphi_{1}\left(\frac{x_{j(1: k) i}}{\sigma}\right)+(k-1) \varphi_{3}\left(\frac{x_{j(1: k) i}}{\sigma}\right)\right)+\sum_{j=\frac{k}{2}+1}^{k}\left(\varphi_{1}\left(\frac{x_{j(k: k) i}}{\sigma}\right)+(k-1) \varphi_{2}\left(\frac{x_{j(k: k) i}}{\sigma}\right)\right)\right\} \\
& +\sum_{i=1}^{m_{3}} \sum_{j=1}^{k}\left\{\varphi_{1}\left(\frac{x_{j(j: j) i}}{\sigma}\right)+(j-1) \varphi_{2}\left(\frac{x_{j(j: j) i}}{\sigma}\right)\right\}+\sum_{i=1}^{m_{4}} \sum_{j=1}^{k}\left\{\varphi_{1}\left(\frac{x_{j(1: j) i}}{\sigma}\right)+(j-1) \varphi_{3}\left(\frac{x_{j(1: j) i}}{\sigma}\right)\right\} \\
& +\sum_{i=1}^{m_{5}}\left\{\sum_{j=1}^{\frac{k}{2}}\left(\varphi_{1}\left(\frac{x_{j\left(\frac{k}{2}: k\right) i}}{\sigma}\right)+\left(\frac{k}{2}-1\right) \varphi_{2}\left(\frac{x_{j\left(\frac{k}{2}: k\right) i}}{\sigma}\right)+\frac{k}{2} \varphi_{3}\left(\frac{x_{j\left(\frac{k}{2}: k\right) i}}{\sigma}\right)\right)\right. \\
& \left.+\sum_{j=\frac{k}{2}+1}^{k}\left(\varphi_{1}\left(\frac{x_{j\left(\frac{k}{2}+1: k\right) i}}{\sigma}\right)+\frac{k}{2} \varphi_{2}\left(\frac{x_{j\left(\frac{k}{2}+1: k\right) i}}{\sigma}\right)+\left(\frac{k}{2}-1\right) \varphi_{3}\left(\frac{x_{j\left(\frac{k}{2}+1: k\right) i}}{\sigma}\right)\right)\right\},
\end{aligned}
$$


where $\varphi_{1}(\cdot)=\log f(\cdot), \varphi_{2}(\cdot)=\log F(\cdot), \varphi_{3}(\cdot)=\log \bar{F}(\cdot)$ and

$$
c=\left\{k ! \prod_{j=1}^{k}\left(\begin{array}{c}
k \\
j
\end{array}\right)\right\}^{m_{1}} k^{m_{2} k}(k !)^{m_{3}+m_{4}}\left\{\frac{1}{B\left(\frac{k}{2}, \frac{k}{2}+1\right)}\right\}^{m_{5} k} .
$$

Denoting the first derivative of $\varphi_{i}(x)$ by $\varphi_{i}^{\prime}(x)$ for $1 \leq i \leq 3$, we get

$$
\begin{aligned}
\frac{\partial l(\sigma)}{\partial \sigma} & =-\sum_{i=1}^{m_{1}} \sum_{j=1}^{k}\left\{\frac{x_{j(j: k) i}}{\sigma^{2}} \varphi_{1}^{\prime}\left(\frac{x_{j(j: k) i}}{\sigma}\right)+(j-1) \frac{x_{j(j: k) i}}{\sigma^{2}} \varphi_{2}^{\prime}\left(\frac{x_{j(j: k) i}}{\sigma}\right)-(k-j) \frac{x_{j(j: k) i}}{\sigma^{2}} \varphi_{3}^{\prime}\left(\frac{x_{j(j: k) i}}{\sigma}\right)\right\} \\
& -\sum_{i=1}^{m_{2}}\left\{\sum_{j=1}^{\frac{k}{2}}\left(\frac{x_{j(1: k) i}}{\sigma^{2}} \varphi_{1}^{\prime}\left(\frac{x_{j(1: k) i}}{\sigma}\right)-(k-1) \frac{x_{j(1: k) i}}{\sigma^{2}} \varphi_{3}^{\prime}\left(\frac{x_{j(1: k) i}}{\sigma}\right)\right)+\sum_{j=\frac{k}{2}+1}^{k}\left(\frac{x_{j(k: k) i}}{\sigma^{2}} \varphi_{1}^{\prime}\left(\frac{x_{j(k: k) i}}{\sigma}\right)\right.\right. \\
& \left.\left.+(k-1) \frac{x_{j(k: k) i}}{\sigma^{2}} \varphi_{2}^{\prime}\left(\frac{x_{j(k: k) i}}{\sigma}\right)\right)\right\}-\sum_{i=1}^{m_{3}} \sum_{j=1}^{k}\left\{\frac{x_{j(j: j) i}}{\sigma^{2}} \varphi_{1}^{\prime}\left(\frac{x_{j(j: j) i}}{\sigma}\right)+(j-1) \frac{x_{j(j: j) i}}{\sigma^{2}} \varphi_{2}^{\prime}\left(\frac{x_{j(j: j) i}}{\sigma}\right)\right\} \\
& -\sum_{i=1}^{m_{4}} \sum_{j=1}^{k}\left\{\frac{x_{j(1: j) i}}{\sigma^{2}} \varphi_{1}^{\prime}\left(\frac{x_{j(1: j) i}}{\sigma}\right)-(j-1) \frac{x_{j(1: j) i}}{\sigma^{2}} \varphi_{3}^{\prime}\left(\frac{x_{j(1: j) i}}{\sigma}\right)\right\}-\sum_{i=1}^{m_{5}}\left\{\sum _ { j = 1 } ^ { \frac { k } { 2 } } \left(\frac{x_{j\left(\frac{k}{2}: k\right) i}}{\sigma^{2}} \varphi_{1}^{\prime}\left(\frac{x_{j\left(\frac{k}{2}: k\right) i}}{\sigma}\right)\right.\right. \\
+ & \left.\left(\frac{k}{2}-1\right) \frac{x_{j\left(\frac{k}{2}: k\right) i}}{\sigma^{2}} \varphi_{2}^{\prime}\left(\frac{x_{j\left(\frac{k}{2}: k\right) i}}{\sigma}\right)-\frac{k}{2} \frac{x_{j\left(\frac{k}{2}: k\right) i}}{\sigma^{2}} \varphi_{3}^{\prime}\left(\frac{x_{j\left(\frac{k}{2}: k\right) i}}{\sigma}\right)\right)+\sum_{j=\frac{k}{2}+1}^{k}\left(\frac{x_{j\left(\frac{k}{2}+1: k\right) i}}{\sigma^{2}} \varphi_{1}^{\prime}\left(\frac{x_{j\left(\frac{k}{2}+1: k\right) i}}{\sigma}\right)\right. \\
+ & \left.\left.\frac{k}{2} \frac{x_{j\left(\frac{k}{2}+1: k\right) i}}{\sigma^{2}} \varphi_{2}^{\prime}\left(\frac{x_{j\left(\frac{k}{2}+1: k\right) i}}{\sigma}\right)-\left(\frac{k}{2}-1\right) \frac{x_{j\left(\frac{k}{2}+1: k\right) i}}{\sigma^{2}} \varphi_{3}^{\prime}\left(\frac{x_{j\left(\frac{k}{2}+1: k\right) i}}{\sigma}\right)\right)\right\}-\frac{M}{\sigma} k .
\end{aligned}
$$

Now, if there exists the MLE of $\sigma$, it satisfies in the equation $\frac{\partial l(\sigma)}{\partial \sigma}=0$. In general, the solution of this equation does not have an explicit form. Nevertheless, we will show the existence and uniqueness of MLEs of scale parameters for the exponential and normal distributions.

\subsection{Exponential distribution}

Let the underlying distribution be exponential with mean $\lambda$. The exponential distribution is a famous member of scale family which is used in different fields such as reliability studies and lifetime data analysis. In the following, the existence and uniqueness of the MLE of the scale parameter is investigated based on the FRSS scheme. Using (2), the likelihood equation can be written as

$$
\begin{aligned}
& -M k \lambda+\sum_{i=1}^{m_{1}} \sum_{j=1}^{k}\left\{(k-j+1) x_{j(j: k) i}-(j-1) \frac{x_{j(j: k) i}}{e^{\frac{x_{j(j: k) i}}{\lambda}}-1}\right\}+\sum_{i=1}^{m_{2}}\left\{\sum_{j=1}^{\frac{k}{2}} k x_{j(1: k) i}\right. \\
& \left.+\sum_{j=\frac{k}{2}+1}^{k}\left[x_{j(k: k) i}-(k-1) \frac{x_{j(k: k) i}}{e^{\frac{x_{j(k: k) i}}{\lambda}}-1}\right]\right\}+\sum_{i=1}^{m_{3}} \sum_{j=1}^{k}\left\{x_{j(j: j) i}-(j-1) \frac{x_{j(j: j) i}}{e^{\frac{x_{j j}(j: j) i}{\lambda}}-1}\right\} \\
& +\sum_{i=1}^{m_{4}} \sum_{j=1}^{k} j x_{j(1: j) i}+\sum_{i=1}^{m_{2}}\left\{\sum_{j=1}^{\frac{k}{2}}\left[\left(\frac{k}{2}-1\right) x_{j\left(\frac{k}{2}: k\right) i}-\left(\frac{k}{2}-1\right) \frac{x_{j\left(\frac{k}{2}: k\right) i}}{e^{\frac{x_{j\left(\frac{k}{2}: k\right) i}}{\lambda}}-1}\right]\right. \\
& +\sum_{j=\frac{k}{2}+1}^{k} \frac{k}{2}\left[x_{j\left(\frac{k}{2}+1: k\right) i}-\frac{x_{j\left(\frac{k}{2}+1: k\right) i}}{\left.\left.e^{\frac{x_{j\left(\frac{k}{2}+1: k\right) i}}{\lambda}}-1\right]\right\}=0 .}\right.
\end{aligned}
$$


Note that the left hand side of equation (3) is a continuous function. On the other hand, by taking $h(\lambda)=\frac{\partial l(\lambda)}{\partial \lambda}$, we have:

$$
\begin{aligned}
\lim _{\lambda \rightarrow 0^{+}} h(\lambda)= & \sum_{i=1}^{m_{1}} \sum_{j=1}^{k}(k-j+1) x_{j(j: k) i}+\sum_{i=1}^{m_{2}}\left[\sum_{j=1}^{\frac{k}{2}} k x_{j(1: k) i}+\sum_{j=\frac{k}{2}+1}^{k} x_{j(k: k) i}\right]+\sum_{i=1}^{m_{3}} \sum_{j=1}^{k} x_{j(j: j) i} \\
& +\sum_{i=1}^{m_{4}} \sum_{j=1}^{k} j x_{j(1: j) i}+\sum_{i=1}^{m_{5}}\left[\sum_{j=1}^{\frac{k}{2}}\left(\frac{k}{2}+1\right) x_{j\left(\frac{k}{2}: k\right) i}+\sum_{j=\frac{k}{2}+1}^{k} \frac{k}{2} x_{j\left(\frac{k}{2}+1: k\right) i}\right]>0,
\end{aligned}
$$

and also $\lim _{\lambda \rightarrow \infty} h(\lambda)<0$. Therefore, the likelihood equation in (3) has at least one solution. On the other hand, note that,

$$
\begin{aligned}
h^{\prime}(\lambda)= & -M k-\frac{1}{\lambda^{2}} \sum_{i=1}^{m_{1}} \sum_{j=1}^{k}(j-1) \frac{x_{j(j: k) i}^{2} e^{\frac{x_{j(j: k) i}}{\lambda}}}{\left(e^{\frac{\left.x_{j(j) k}\right) i}{\lambda}}-1\right)^{2}}-\frac{1}{\lambda^{2}} \sum_{i=1}^{m_{2}} \sum_{j=\frac{k}{2}+1}^{k}(k-1) \frac{x_{j(k: k) i}^{2} e^{\frac{x_{j(k: k) i}}{\lambda}}}{\left(e^{\frac{x_{j(k: k) i}}{\lambda}}-1\right)^{2}} \\
& -\frac{1}{\lambda^{2}} \sum_{i=1}^{m_{3}} \sum_{j=1}^{k}(j-1) \frac{x_{j(j: j) i}^{2} e^{\frac{x_{j(j ; j) i}}{\lambda}}}{\left(e^{\frac{x_{j(j ; j) i}}{\lambda}}-1\right)^{2}}-\frac{1}{\lambda^{2}} \sum_{i=1}^{m_{5}} \sum_{j=1}^{\frac{k}{2}}\left(\frac{k}{2}-1\right) \frac{x_{j\left(\frac{k}{2}: k\right) i}^{2} e^{\frac{x_{j\left(\frac{k}{2}: k\right) i}}{\lambda}}}{\left(e^{\frac{x_{\left(\frac{k}{2}: k\right) i}^{2}}{\lambda}}-1\right)^{2}} \\
& -\frac{1}{\lambda^{2}} \sum_{i=1}^{m_{5}} \sum_{j=\frac{k}{2}+1}^{k} \frac{k}{2} \frac{x_{j\left(\frac{k}{2}+1: k\right) i}^{2} e^{\frac{x_{j\left(\frac{k}{2}+1: k\right) i}}{\lambda}}}{\left(e^{\frac{x_{j\left(\frac{k}{2}+1: k\right) i}}{\lambda}}-1\right)^{2}}
\end{aligned}
$$

which is simply a negative expression for all $\lambda$. Hence, the solution of the equation (3) and consequently the MLE of $\lambda$ is unique, too.

In the next subsection, we investigate the existence and uniqueness of the MLE for scale parameter in normal distribution using $\mathbf{X}_{F R S S}$.

\subsection{Normal distribution}

Suppose that a FRSS data set is collected from the normal distribution with mean zero and variance $\sigma^{2}$. Using (2), the MLE of $\sigma$ is obtained by solving the following equation

$$
\begin{aligned}
& \sum_{i=1}^{m_{1}} \sum_{j=1}^{k} \frac{x_{j(j: k) i}}{\sigma}\left\{\frac{x_{j(j: k) i}}{\sigma}-(j-1) \frac{\phi\left(\frac{x_{j(j: k) i}}{\sigma}\right)}{\Phi\left(\frac{x_{j(j: k) i}}{\sigma}\right)}+(k-j) \frac{\phi\left(\frac{x_{j(j: k) i}}{\sigma}\right)}{\Phi\left(-\frac{\left.x_{j(j: k) i}\right)}{\sigma}\right)}\right\}+\sum_{i=1}^{m_{2}} \sum_{j=1}^{\frac{k}{2}} \frac{x_{j(1: k) i}}{\sigma} \\
& \times\left\{\frac{x_{j(1: k) i}}{\sigma}+(k-1) \frac{\phi\left(\frac{x_{j(1: k) i}}{\sigma}\right)}{\Phi\left(-\frac{x_{j(1: k) i}}{\sigma}\right)}\right\}+\sum_{i=1}^{m_{2}} \sum_{j=\frac{k}{2}+1}^{k} \frac{x_{j(k: k) i}}{\sigma}\left\{\frac{x_{j(k: k) i}}{\sigma}-(k-1) \frac{\phi\left(\frac{x_{j(k: k) i}}{\sigma}\right)}{\Phi\left(\frac{x_{j(k: k) i}}{\sigma}\right)}\right\} \\
& +\sum_{i=1}^{m_{3}} \sum_{j=1}^{k}\left\{\frac{x_{j(j: j) i}}{\sigma}-(j-1) \frac{x_{j(j: j) i}}{\sigma} \frac{\phi\left(\frac{x_{j(j: j) i}}{\sigma}\right)}{\Phi\left(\frac{\left.x_{j(j: j) i}\right)}{\sigma}\right)}\right\}+\sum_{i=1}^{m_{4}} \sum_{j=1}^{k} \frac{x_{j(1: j) i}}{\sigma}\left\{\frac{x_{j(1: j) i}}{\sigma}+(j-1) \frac{\phi\left(\frac{x_{j(1: j) i}}{\sigma}\right)}{\Phi\left(-\frac{\left.x_{j(1: j) i}\right)}{\sigma}\right)}\right\} \\
& +\sum_{i=1}^{m_{5}} \sum_{j=1}^{\frac{k}{2}} \frac{x_{j\left(\frac{k}{2}: k\right) i}}{\sigma}\left\{\frac{x_{j\left(\frac{k}{2}: k\right) i}}{\sigma}-\left(\frac{k}{2}-1\right) \frac{\phi\left(\frac{x_{j\left(\frac{k}{2}: k\right) i}}{\sigma}\right)}{\Phi\left(\frac{x_{j\left(\frac{k}{2}: k\right) i}}{\sigma}\right)}+\frac{k}{2} \frac{\phi\left(\frac{x_{j\left(\frac{k}{2}: k\right) i}}{\sigma}\right)}{\Phi\left(-\frac{x_{j\left(\frac{k}{2}: k\right) i}}{\sigma}\right)}\right\} \\
& +\sum_{i=1}^{m_{5}} \sum_{j=\frac{k}{2}+1}^{k} \frac{x_{j\left(\frac{k}{2}+1: k\right) i}}{\sigma}\left\{\frac{x_{j\left(\frac{k}{2}+1: k\right) i}}{\sigma}-\frac{k}{2} \frac{\phi\left(\frac{x_{j\left(\frac{k}{2}+1: k\right) i}}{\sigma}\right)}{\Phi\left(\frac{x_{j\left(\frac{k}{2}+1: k\right) i}}{\sigma}\right)}+\left(\frac{k}{2}-1\right) \frac{\phi\left(\frac{x_{j\left(\frac{k}{2}+1: k\right) i}}{\sigma}\right)}{\Phi\left(-\frac{x_{j\left(\frac{k}{2}+1: k\right) i}}{\sigma}\right)}\right\}-M k=0,
\end{aligned}
$$

where $\phi(\cdot)$ and $\Phi(\cdot)$ stand for the pdf and cdf of the standard normal distribution. Note that the left hand side of (4) is a continuous function with respect to $\sigma$, and converges to $\infty$ and $(-M k)$, a negative value, when $\sigma$ tends to 
0 and $\infty$, respectively. Hence, the Eq. (4) has at least one solution. To investigate the uniqueness of the MLE, we have

$$
\frac{\partial^{2} l(\sigma)}{\partial \sigma^{2}}=C+D_{1}+D_{2}+D_{3}
$$

where

$$
\begin{aligned}
& -\frac{\sigma^{2}}{2} C=-M k+\sum_{i=1}^{m_{1}} \sum_{j=1}^{k} \frac{x_{j(j: k) i}}{\sigma}\left\{\frac{x_{j(j: k) i}}{\sigma}-(j-1) \frac{\phi\left(\frac{x_{j(j: k) i}}{\sigma}\right)}{\Phi\left(\frac{x_{j(j: k) i}}{\sigma}\right)}+(k-j) \frac{\phi\left(\frac{x_{j(j: k) i}}{\sigma}\right)}{\Phi\left(-\frac{x_{j(j: k) i}}{\sigma}\right)}\right\} \\
& +\sum_{i=1}^{m_{2}} \sum_{j=1}^{\frac{k}{2}} \frac{x_{j(1: k) i}}{\sigma}\left\{\frac{x_{j(1: k) i}}{\sigma}+(k-1) \frac{\phi\left(\frac{x_{j(1: k) i}}{\sigma}\right)}{\Phi\left(-\frac{x_{j(1: k) i}}{\sigma}\right)}\right\}+\sum_{i=1}^{m_{2}} \sum_{j=\frac{k}{2}+1}^{k} \frac{x_{j(k: k) i}}{\sigma}\left\{\frac{x_{j(k: k) i}}{\sigma}\right. \\
& \left.-(k-1) \frac{\phi\left(\frac{x_{j(k: k) i}}{\sigma}\right)}{\Phi\left(\frac{x_{j(k: k) i}}{\sigma}\right)}\right\}+\sum_{i=1}^{m_{3}} \sum_{j=1}^{k} \frac{x_{j(j: j) i}}{\sigma}\left\{\frac{x_{j(j: j) i}}{\sigma}-(j-1) \frac{\phi\left(\frac{x_{j(j: j) i}}{\sigma}\right)}{\Phi\left(\frac{x_{j(j: j) i}}{\sigma}\right)}\right\} \\
& +\sum_{i=1}^{m_{4}} \sum_{j=1}^{k} \frac{x_{j(1: j) i}}{\sigma}\left\{(j-1) \frac{\phi\left(\frac{x_{j(1: j) i}}{\sigma}\right)}{\Phi\left(-\frac{x_{j(1: j) i}}{\sigma}\right)}+\frac{x_{j(1: j) i}}{\sigma}\right\} \\
& +\sum_{i=1}^{m_{5}} \sum_{j=1}^{\frac{k}{2}} \frac{x_{j\left(\frac{k}{2}: k\right) i}}{\sigma}\left\{\frac{x_{j\left(\frac{k}{2}: k\right) i}}{\sigma}-\left(\frac{k}{2}-1\right) \frac{\phi\left(\frac{x_{j\left(\frac{k}{2}: k\right) i}}{\sigma}\right)}{\Phi\left(\frac{x_{j\left(\frac{k}{2}: k\right) i}}{\sigma}\right)}+\frac{k}{2} \frac{\phi\left(\frac{x_{j\left(\frac{k}{2}: k\right) i}}{\sigma}\right)}{\Phi\left(-\frac{x_{j\left(\frac{k}{2}: k\right) i}}{\sigma}\right)}\right\} \\
& +\sum_{i=1}^{m_{5}} \sum_{j=\frac{k}{2}+1}^{k} \frac{x_{j\left(\frac{k}{2}+1: k\right) i}}{\sigma}\left\{\frac{x_{j\left(\frac{k}{2}+1: k\right) i}}{\sigma}-\frac{k}{2} \frac{\phi\left(\frac{x_{j\left(\frac{k}{2}+1: k\right) i}}{\sigma}\right)}{\Phi\left(\frac{x_{j\left(\frac{k}{2}+1: k\right) i}}{\sigma}\right)}+\left(\frac{k}{2}-1\right) \frac{\phi\left(\frac{x_{j\left(\frac{k}{2}+1: k\right) i}}{\sigma}\right)}{\Phi\left(-\frac{x_{j\left(\frac{k}{2}+1: k\right) i}}{\sigma}\right)}\right\}, \\
& -\sigma^{2} D_{1}=\sum_{i=1}^{m_{1}} \sum_{j=1}^{k} \frac{x_{j(j: k) i}^{2}}{\sigma^{2}}+\sum_{i=1}^{m_{2}}\left(\sum_{j=1}^{\frac{k}{2}} \frac{x_{j(1: k) i}^{2}}{\sigma^{2}}+\sum_{j=\frac{k}{2}+1}^{k} \frac{x_{j(k: k) i}^{2}}{\sigma^{2}}\right)+\sum_{i=1}^{m_{3}} \sum_{j=1}^{k} \frac{x_{j(j: j) i}^{2}}{\sigma^{2}} \\
& +\sum_{i=1}^{m_{4}} \sum_{j=1}^{k} \frac{x_{j(1: j) i}^{2}}{\sigma^{2}}+\sum_{i=1}^{m_{5}}\left(\sum_{j=1}^{\frac{k}{2}} \frac{x_{j\left(\frac{k}{2}: k\right) i}^{2}}{\sigma^{2}}+\sum_{j=\frac{k}{2}+1}^{k} \frac{x_{j\left(\frac{k}{2}+1: k\right) i}^{2}}{\sigma^{2}}\right)+M k, \\
& -\sigma^{2} D_{2}=\sum_{i=1}^{m_{1}} \sum_{j=1}^{k}(j-1) \frac{x_{j(j: k) i}^{2}}{\sigma^{2}} \frac{\phi\left(\frac{x_{j(j: k) i}}{\sigma}\right)}{\Phi\left(\frac{x_{j(j: k) i}}{\sigma}\right)}\left(\frac{x_{j(j: k) i}}{\sigma}+\frac{\phi\left(\frac{x_{j(j: k) i}}{\sigma}\right)}{\Phi\left(\frac{x_{j(j: k) i}}{\sigma}\right)}\right)+\sum_{i=1}^{m_{2}} \sum_{j=\frac{k}{2}+1}^{k}(k-1) \frac{x_{j(k: k) i}^{2}}{\sigma^{2}} \\
& \times \frac{\phi\left(\frac{x_{j(k: k) i}}{\sigma}\right)}{\Phi\left(\frac{x_{j(k: k) i}}{\sigma}\right)}\left(\frac{x_{j(k: k) i}}{\sigma}+\frac{\phi\left(\frac{x_{j(k: k) i}}{\sigma}\right)}{\Phi\left(\frac{x_{j(k: k) i}}{\sigma}\right)}\right)+\sum_{i=1}^{m_{3}} \sum_{j=1}^{k}(j-1) \frac{x_{j(j: j) i}^{2}}{\sigma^{2}} \frac{\phi\left(\frac{x_{j(j: j) i}}{\sigma}\right)}{\Phi\left(\frac{x_{j(j: j) i}}{\sigma}\right)} \\
& \times\left(\frac{x_{j(j: j) i}}{\sigma}+\frac{\phi\left(\frac{x_{j(j: j) i}}{\sigma}\right)}{\Phi\left(\frac{\left.x_{j(j: j) i}\right)}{\sigma}\right)}\right)+\sum_{i=1}^{m_{5}} \sum_{j=1}^{\frac{k}{2}}\left(\frac{k}{2}-1\right)\left(\frac{x_{j\left(\frac{k}{2}: k\right) i}}{\sigma}+\frac{\phi\left(\frac{x_{j\left(\frac{k}{2}: k\right) i}}{\sigma}\right)}{\Phi\left(\frac{x_{j\left(\frac{k}{2}: k\right) i}}{\sigma}\right)}\right) \frac{x_{j\left(\frac{k}{2}: k\right) i}^{2}}{\sigma^{2}} \frac{\phi\left(\frac{x_{j\left(\frac{k}{2}: k\right) i}}{\sigma}\right)}{\Phi\left(\frac{x_{j\left(\frac{k}{2}: k\right) i}}{\sigma}\right)} \\
& +\sum_{i=1}^{m_{5}} \sum_{j=\frac{k}{2}+1}^{k}\left(\frac{k}{2} \frac{x_{j\left(\frac{k}{2}+1: k\right) i}^{2}}{\sigma^{2}} \frac{\phi\left(\frac{x_{j\left(\frac{k}{2}+1: k\right) i}}{\sigma}\right)}{\Phi\left(\frac{x_{j\left(\frac{k}{2}+1: k\right) i}}{\sigma}\right)}\left(\frac{x_{j\left(\frac{k}{2}+1: k\right) i}}{\sigma}+\frac{\phi\left(\frac{x_{j\left(\frac{k}{2}+1: k\right) i}}{\sigma}\right)}{\Phi\left(\frac{\left.x_{j\left(\frac{k}{2}+1: k\right) i}\right)}{\sigma}\right)}\right)\right.
\end{aligned}
$$


and

$$
\begin{aligned}
& \sigma^{2} D_{3}=\sum_{i=1}^{m_{1}} \sum_{j=1}^{k}(k-j) \frac{x_{j(j: k) i}^{2}}{\sigma^{2}} \frac{\phi\left(\frac{x_{j(j: k) i}}{\sigma}\right)}{\Phi\left(-\frac{x_{j(j: k) i}}{\sigma}\right)}\left(\frac{x_{j(j: k) i}}{\sigma}-\frac{\phi\left(\frac{x_{j(j: k) i}}{\sigma}\right)}{\Phi\left(-\frac{x_{j(j: k) i}}{\sigma}\right)}\right) \\
& +\sum_{i=1}^{m_{2}} \sum_{j=1}^{\frac{k}{2}}(k-1) \frac{x_{j(1: k) i}^{2}}{\sigma^{2}} \frac{\phi\left(\frac{x_{j(1: k) i}}{\sigma}\right)}{\Phi\left(-\frac{x_{j(1: k) i}}{\sigma}\right)}\left(\frac{x_{j(1: k) i}}{\sigma}-\frac{\phi\left(\frac{x_{j(1: k) i}}{\sigma}\right)}{\Phi\left(-\frac{x_{j(1: k) i}}{\sigma}\right)}\right) \\
& +\sum_{i=1}^{m_{4}} \sum_{j=1}^{k}(j-1)\left(\frac{x_{j(1: j) i}}{\sigma}-\frac{\phi\left(\frac{x_{j(1: j) i}}{\sigma}\right)}{\Phi\left(-\frac{x_{j(1: j) i}}{\sigma}\right)}\right) \frac{x_{j(1: j) i}^{2}}{\sigma^{2}} \frac{\phi\left(\frac{x_{j(1: j) i}}{\sigma}\right)}{\Phi\left(-\frac{x_{j(1: j) i}}{\sigma}\right)} \\
& +\sum_{i=1}^{m_{5}} \sum_{j=1}^{\frac{k}{2}} \frac{k}{2} \frac{x_{j\left(\frac{k}{2}: k\right) i}^{2}}{\sigma^{2}} \frac{\phi\left(\frac{x_{j\left(\frac{k}{2}: k\right) i}}{\sigma}\right)}{\Phi\left(-\frac{x_{j\left(\frac{k}{2}: k\right) i}}{\sigma}\right)}\left(\frac{x_{j\left(\frac{k}{2}: k\right) i}}{\sigma}-\frac{\phi\left(\frac{x_{j\left(\frac{k}{2}: k\right) i}}{\sigma}\right)}{\Phi\left(-\frac{x_{j\left(\frac{k}{2}: k\right) i}}{\sigma}\right)}\right) \\
& \left.+\sum_{i=1}^{m_{5}} \sum_{j=\frac{k}{2}+1}^{k}\left(\frac{k}{2}-1\right) \frac{x_{j\left(\frac{k}{2}+1: k\right) i}^{2}}{\sigma^{2}} \frac{\phi\left(\frac{x_{j\left(\frac{k}{2}+1: k\right) i}}{\sigma}\right)}{\Phi\left(-\frac{x_{j\left(\frac{k}{2}+1: k\right) i}}{\sigma}\right)} \frac{x_{j\left(\frac{k}{2}+1: k\right) i}}{\sigma}-\frac{\phi\left(\frac{x_{j\left(\frac{k}{2}+1: k\right) i}}{\sigma}\right)}{\Phi\left(-\frac{x_{j\left(\frac{k}{2}+1: k\right) i}}{\sigma}\right)}\right) .
\end{aligned}
$$

The expression in the right hand side of (5) is exactly the same as the one in the left hand side of (4) and so, it is equal to zero at any root of the Eq. (4). On the other hand, it is easily seen that $D_{1}$ in (6) is always negative. Also, since $\left(x+\frac{\phi(x)}{\Phi(x)}\right)>0$ and $\left(x-\frac{\phi(x)}{\Phi(-x)}\right)<0$ for all $x$ (see, for example, 9), the expressions $D_{2}$ and $D_{3}$ in (7) and (8), respectively, are negative. Hence, the second derivative of the likelihood function is always negative. These imply that the MLE of $\sigma$ is unique.

\section{Fisher information in FRSS}

In this section, the FI contained in a FRSS data set is studied. It is easy to show that under regularity conditions (see, 11, p.68), the amount of FI in a FRSS data set about a vector of parameters $\boldsymbol{\theta}$ equals to

$$
I_{F R S S}(\boldsymbol{\theta})=I_{R S S}(\boldsymbol{\theta})+I_{E R S S}(\boldsymbol{\theta})+I_{M E R S S-I}(\boldsymbol{\theta})+I_{M E R S S-I I}(\boldsymbol{\theta})+I_{M R S S}(\boldsymbol{\theta}) .
$$

Let $F(\cdot ; \boldsymbol{\theta})$ be the cdf of the underlying population. [7] showed that the FI matrix contained in a RSS data set is given by

$$
I_{R S S}(\boldsymbol{\theta})=I_{S R S}(\boldsymbol{\theta})+m_{1} k(k-1) E\left\{\frac{1}{F(X ; \boldsymbol{\theta}) \bar{F}(X ; \boldsymbol{\theta})} \frac{\partial}{\partial \boldsymbol{\theta}} F(X ; \boldsymbol{\theta}) \frac{\partial}{\partial \boldsymbol{\theta}^{T}} F(X ; \boldsymbol{\theta})\right\},
$$

where $I_{S R S}(\cdot)$ stands for the FI matrix in a SRS. [15] compared the FI about the dependence parameter using RSS with SRS. [9] investigated the FI in MERSS procedure for a scale family of distributions.

Although, there is not a closed form for the FI in general, but we can obtain the FI for special case of exponential distribution.

\section{Remark 1}

[5, p. 166] Let $X_{1}, \cdots, X_{k}$ be a random sample from an exponential distribution with mean $\lambda$. Then, the FI contained in the $j$ th order statistic, denoted by $I_{j: k}(\lambda)$, is given by

$$
I_{j: k}(\lambda)= \begin{cases}\frac{1}{\lambda^{2}}, & j=1, \\ \frac{1}{\lambda^{2}}+\frac{2 k(k-1)}{\lambda^{2}} \zeta(3, k), & j=2, \\ \frac{1}{\lambda^{2}}+\frac{k(k-j+1)}{\lambda^{2}(j-2)}\left(\gamma_{j, k}+\delta_{j, k}\right), & j \geq 3,\end{cases}
$$

where $\zeta(\alpha, r)=\sum_{i=0}^{\infty} \frac{1}{(r+i)^{\alpha}}, \gamma_{j, k}=\left[\sum_{i=1}^{j-2} \frac{1}{k-i}\right]^{2}$ and $\delta_{j, k}=\sum_{i=1}^{j-2} \frac{1}{(k-i)^{2}}$. 
The FI in various schemes can be obtained using Remark 1. Of course, the FI in RSS procedure may be computed using (10). For this purpose, we have

$$
\begin{aligned}
I_{R S S}(\lambda) & =\frac{m_{1} k}{\lambda^{2}}+\frac{m_{1} k(k-1)}{\lambda^{5}} \int_{0}^{\infty} \frac{x^{2} e^{-\frac{2 x}{\lambda}}}{1-e^{-\frac{x}{\lambda}}} d x \\
& =\frac{m_{1} k}{\lambda^{2}}+\frac{m_{1} k(k-1)}{\lambda^{5}} \sum_{i=0}^{\infty} \int_{0}^{\infty} x^{2} e^{-(i+2) \frac{x}{\lambda}} d x \\
& =\frac{m_{1} k}{\lambda^{2}}+\frac{m_{1} k(k-1)}{\lambda^{2}} \zeta(3,2) \\
& =\frac{m_{1} k}{\lambda^{2}}[1+(k-1) \zeta(3,2)] .
\end{aligned}
$$

Now, using Remark 1, it can be shown that the FI in ERSS, MERSS type I, MERSS type II and MRSS schemes are, respectively, as follows

$$
\begin{aligned}
& I_{E R S S}(\lambda)=\frac{m_{2} k}{2}\left[I_{1: k}(\lambda)+I_{k: k}(\lambda)\right] \\
&= \frac{m_{2} k}{2}\left[\frac{1}{\lambda^{2}}+\frac{1}{\lambda^{2}}+\frac{k}{\lambda^{2}(k-2)}\left(\gamma_{k, k}+\delta_{k, k}\right)\right] \\
&= \frac{m_{2} k}{2 \lambda^{2}}\left[2+\frac{k}{k-2}\left(\gamma_{k, k}+\delta_{k, k}\right)\right], \\
& I_{M E R S S-I}(\lambda)=m_{3}\left\{\frac{1}{\lambda^{2}}+I_{2: 2}(\lambda)+\sum_{j=3}^{k} I_{j: j}(\lambda)\right\} \\
&=m_{3}\left\{\frac{2}{\lambda^{2}}+\frac{4}{\lambda^{2}} \zeta(3,2)+\sum_{j=3}^{k}\left[\frac{1}{\lambda^{2}}+\frac{j}{\lambda^{2}(j-2)}\left(\gamma_{j, j}+\delta_{j, j}\right)\right]\right\} \\
&=\frac{m_{3}}{\lambda^{2}}\left\{r+4 \zeta(3,2)+\sum_{j=3}^{k} \frac{j}{j-2}\left[\gamma_{j, j}+\delta_{j, j}\right]\right\} \\
& I_{M E R S S-I I}(\lambda)=m_{4} \sum_{j=1}^{k} I_{1: j}(\lambda)=\frac{m_{4} k}{\lambda^{2}},
\end{aligned}
$$

and

$$
I_{M R S S}(\lambda)=\frac{m_{5} k}{2}\left\{I_{\frac{k}{2}: k}(\lambda)+I_{\frac{k}{2}+1: k}(\lambda)\right\}
$$

where

$$
I_{\frac{k}{2}: k}(\lambda)= \begin{cases}\frac{1}{\lambda^{2}}+\frac{2 k(k-1)}{\lambda^{2}} \zeta(3, k), & \frac{k}{2}=2, \\ \frac{1}{\lambda^{2}}+\frac{k(k / 2+1)}{\lambda^{2}(k / 2-2)}\left[\gamma_{\frac{k}{2}, k}+\delta_{\frac{k}{2}, k}\right], & \frac{k}{2} \geq 3 .\end{cases}
$$

Consequently, the FI contained in $\mathbf{X}_{F R S S}^{(M)}$ about $\lambda$ is obtained by summing Eqs. (11)-(15) as

$$
I_{F R S S}(\lambda)=I_{R S S}(\lambda)+I_{E R S S}(\lambda)+I_{M E R S S-I}(\lambda)+I_{M E R S S-I I}(\lambda)+I_{M R S S}(\lambda) .
$$

The following result is concluded as trivial using the asymptotic properties of the MLE. 
Table 1. Values of FI of some selected permutations of $\mathbf{m}$ for exponential distribution.

\begin{tabular}{|c|c|c|c|c|c|c|c|}
\hline \multicolumn{4}{|c|}{$k=4$} & \multicolumn{4}{|c|}{$k=6$} \\
\hline \multicolumn{2}{|c|}{ The most FIs } & \multicolumn{2}{|c|}{ The least FIs } & \multicolumn{2}{|c|}{ The most FIs } & \multicolumn{2}{|c|}{ The least FIs } \\
\hline $\mathbf{m}$ & FI & $\mathbf{m}$ & FI & $\mathbf{m}$ & FI & $\mathbf{m}$ & FI \\
\hline$(0,0,0,0,6)$ & 56.85905 & $(1,3,0,1,1)$ & 46.99254 & $(0,0,0,0,6)$ & 119.97000 & $(0,4,0,2,0)$ & 73.99000 \\
\hline$(1,0,0,0,5)$ & 56.23191 & $(0,3,1,1,1)$ & 46.56251 & $(1,0,0,0,5)$ & 118.09841 & $(0,3,0,3,0)$ & 64.49250 \\
\hline$(0,0,1,0,5)$ & 55.80188 & $(0,4,0,1,1)$ & 46.36540 & $(2,0,0,0,4)$ & 116.22683 & $(0,1,0,4,1)$ & 59.49250 \\
\hline$(2,0,0,0,4)$ & 55.60477 & $(1,3,1,1,0)$ & 45.93537 & $(0,0,1,0,5)$ & 116.22221 & $(1,1,0,4,0)$ & 57.62091 \\
\hline$(1,0,1,0,4)$ & 55.17474 & $(0,2,0,4,0)$ & 32.44444 & $(0,1,0,0,5)$ & 115.47250 & $(0,2,0,4,0)$ & 54.99500 \\
\hline$(3,0,0,0,3)$ & 54.97762 & $(0,0,0,5,1)$ & 29.47651 & $(3,0,0,0,3)$ & 114.35524 & $(0,0,0,5,1)$ & 49.99500 \\
\hline$(6,0,0,0,0)$ & 53.09619 & $(1,0,0,5,0)$ & 28.84937 & $(6,0,0,0,0)$ & 108.74049 & $(1,0,0,5,0)$ & 48.12341 \\
\hline$(5,0,1,0,0)$ & 52.66617 & $(0,0,1,5,0)$ & 28.41934 & $(4,0,1,0,1)$ & 108.73587 & $(0,0,1,5,0)$ & 46.24721 \\
\hline$(5,1,0,0,0)$ & 52.46905 & $(0,1,0,5,0)$ & 28.22222 & $(2,0,2,0,2)$ & 108.73125 & $(0,1,0,5,0)$ & 45.49750 \\
\hline$(4,0,2,0,0)$ & 52.23614 & $(0,0,0,6,0)$ & 24.00000 & $(5,0,1,0,0)$ & 106.86428 & $(0,0,0,6,0)$ & 36.00000 \\
\hline
\end{tabular}

\section{Remark 2}

Let $\hat{\lambda}_{F R S S}$ be the MLE of $\lambda$ based on the FRSS scheme with $M$ cycles. Then the asymptotic distribution of $\hat{\lambda}_{F R S S}$ is given by

$$
\sqrt{n}\left(\hat{\lambda}_{F R S S}-\lambda\right) \rightarrow N\left(0, \frac{1}{I_{F R S S}(\lambda)}\right), \quad \text { as } n \rightarrow \infty,
$$

where $n=M k$ is the size of measured data.

Since the first and second derivatives of log-likelihood function in normal distribution have complex functional forms, we can not derive a closed form for the FI in this case. So, numerical methods are needed to compute it.

The FIs are calculated for all permutations of $\mathbf{m}=\left(m_{1}, m_{2}, m_{3}, m_{4}, m_{5}\right)$. It is obvious that the number of all permutations of $\mathbf{m}=\left(m_{1}, m_{2}, m_{3}, m_{4}, m_{5}\right)$ for $M=6$ equals 210 , so, we have just presented 10 permutations with the most FIs and also 10 permutations with the least FIs in Table 1.

From Table 1, the following outcomes deduced:

- The FI increases, when $n=M K$ increases for all permutations of $\mathbf{m}=\left(m_{1}, m_{2}, m_{3}, m_{4}, m_{5}\right)$.

- The schemes $(0,0,0,0,6)$ and $(0,0,0,6,0)$ have maximum and minimum values of FI, respectively.

- One can derive the most FIs between all permutations of $\mathbf{m}=\left(m_{1}, m_{2}, m_{3}, m_{4}, m_{5}\right)$ by Combining MRSS and RSS schemes. Furthermore, combinations of MERSS type-II and ERSS schemes have the least FIs in among all permutations. 


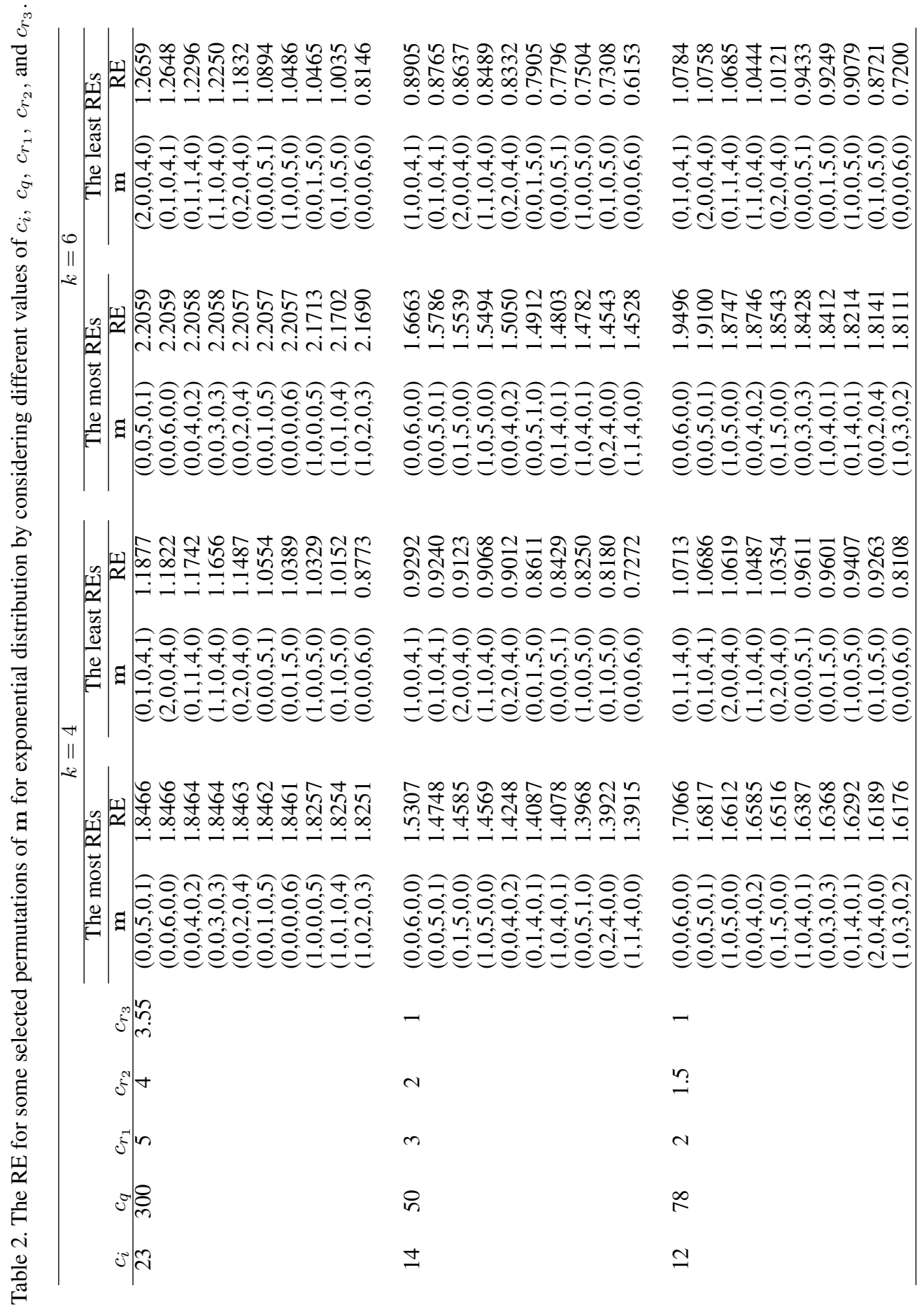




\section{Optimal FRSS scheme}

In this section, the optimal scheme $\mathbf{m}=\left(m_{1}, m_{2}, m_{3}, m_{4}, m_{5}\right)$ is determined via defining a RE criterion. Toward this end, we use two separate criteria, the efficiency of estimators and the costs of sampling. The idea of 18 has been used in this section. It should be highlighted that on the basis of philosophy of RSS scheme, there are different costs such as cost of sampling one unit $\left(c_{i}\right)$, cost of quantification of the interested variable for one unit $\left(c_{q}\right)$, cost of one pairwise comparison in RSS and MRSS schemes $\left(c_{r_{1}}\right)$, cost of one pairwise comparison in ERSS scheme $\left(c_{r_{2}}\right)$ and cost of one pairwise comparison in MERSS type I and MERSS type II schemes $c_{r_{3}}$. It is worth pointing out that from the philosophy of different mentioned schemes of RSS in this paper, one can easily find out that $c_{i}$ is less than $c_{q}$ and $c_{r_{3}} \leq c_{r_{2}} \leq c_{r_{1}}$. Therefore, the total cost of FRSS scheme can be computed as

$$
\begin{aligned}
C_{F R S S}= & k\left(m_{1}+m_{5}\right)\left[k c_{i}+g_{1}(k) c_{r_{1}}+c_{q}\right]+m_{2} k\left(k c_{i}+g_{2}(k) c_{r_{2}}+c_{q}\right) \\
& +\left(m_{3}+m_{4}\right)\left[\frac{k(k+1)}{2} c_{i}+k\left(g_{2}(k) c_{r_{3}}+c_{q}\right)\right],
\end{aligned}
$$

where $g_{1}(k) \approx \frac{(k+2)(k-1)}{2}$ stands for the number of needed pairwise comparisons for judgment ranking in both of RSS and MRSS schemes; also, $g_{2}(k) \approx k-1$ represents the number of needed pairwise comparisons for judgment ranking in the ERSS, MERSS type I and MERSS type II schemes. Moreover, the total cost of SRS is given by

$$
C_{S R S}=N\left(c_{i}+c_{q}\right) .
$$

Now, suppose that $T_{F R S S}$ and $T_{S R S}$ are two estimators based on the FRSS and SRS schemes, respectively. Then, the RE of $T_{F R S S}$ with respect to $T_{S R S}$ is defined as

$$
\operatorname{RE}\left(T_{F R S S}, T_{S R S}\right)=\frac{C_{S R S}}{C_{F R S S}} \frac{\operatorname{MSE}\left(T_{S R S}\right)}{\operatorname{MSE}\left(T_{F R S S}\right)},
$$

where $\operatorname{MSE}(T)=E(T-\theta)^{2}$ stands for the mean squared error of the estimator $T$ of the parameter $\theta$. For more details, see also, [24]. In the sequel, we compare the REs of MLEs of the scale parameters of both exponential and normal distributions based on the FRSS and SRS schemes with same size $n=M k$.

\subsection{Exponential distribution}

Based on a simple random sample from the exponential distribution with mean $\lambda$, it can be easily shown that the MLE of $\lambda$ is the sample mean, that is, $\hat{\lambda}_{S R S}=\bar{X}$, and it is easy to see that $\hat{\lambda}_{S R S}$ is an unbiased estimator with variance $\lambda^{2} / n$. On the other hand, using Remark 2, for large values of $n$, it is clear that approximately, $\operatorname{Var}\left(\hat{\lambda}_{F R S S}\right)=\left(n I_{F R S S}(\lambda)\right)^{-1}$. Therefore, one can obtain the $\operatorname{RE}\left(\hat{\lambda}_{F R S S}, \hat{\lambda}_{S R S}\right)$ using (17). Toward this end, some different costs have been considered. The REs are computed for $M=6$ and $k=4,6$. We have just presented 10 permutations with the most REs and also 10 permutations with the least REs in Table 2.

From Table 2 the following outcomes can be deduced:

- The estimator $\hat{\lambda}_{F R S S}$ is more efficient than $\hat{\lambda}_{S R S}$ for many permutations of $\mathbf{m}=\left(m_{1}, m_{2}, m_{3}, m_{4}, m_{5}\right)$.

- The schemes $(0,0,5,0,1),(0,0,6,0,0)$ and $(0,0,4,0,2)$ are common in Table 2 among 10 permutations which have the most REs. So, one of them may be considered as the optimal FRSS scheme for estimating the mean of exponential distribution.

- Using the entries of Table 2, the RE of different schemes in the problem of estimating the mean of exponential distribution may also be compared together. For example, when $k=4$, the RE of MERSS type I, the case of $\mathbf{m}=(0,0,6,0,0)$, with respect to MERSS type II, the case of $\mathbf{m}=(0,0,0,6,0)$, is $1.8466 / 0.8773=2.1049$, which implies that MERSS type I is more efficient than MERSS type II.

- The RE is sensitive to changes of the cost values and permutations order of $\mathbf{m}=\left(m_{1}, m_{2}, m_{3}, m_{4}, m_{5}\right)$ is changed by different values of costs. 


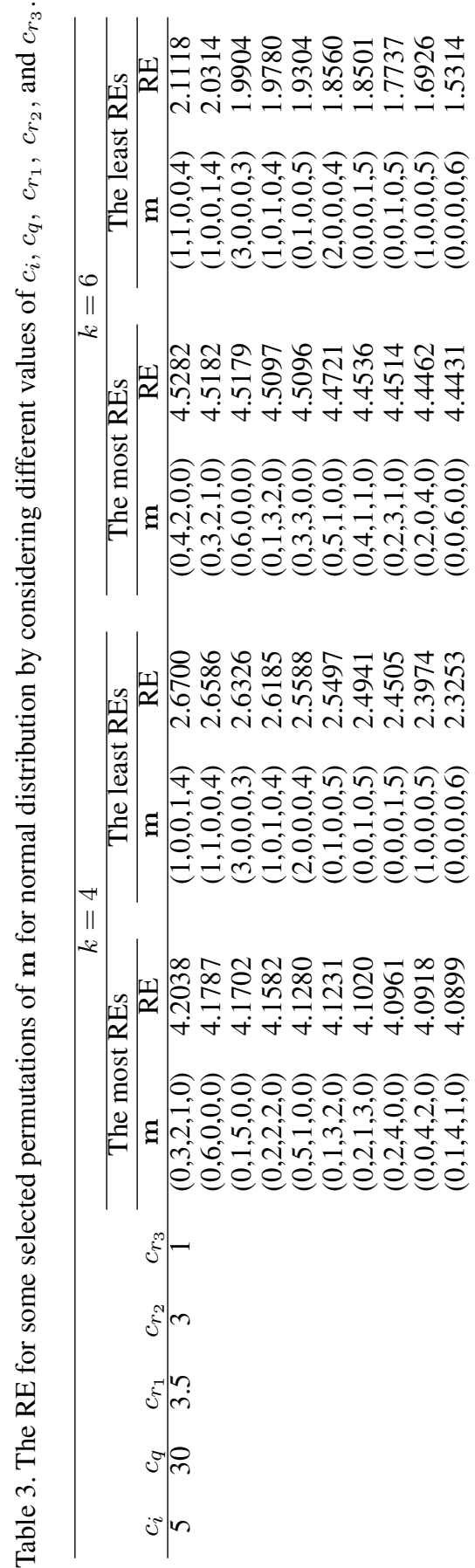

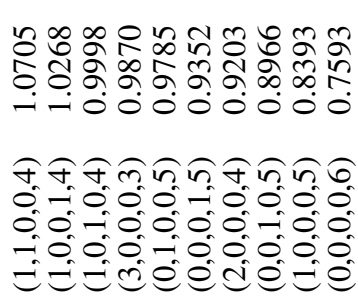

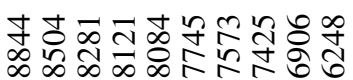

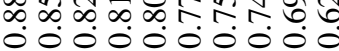

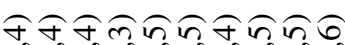

$0=0.000$

0.00

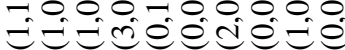

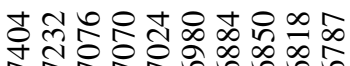

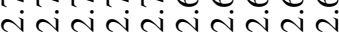

츠유윰ำ

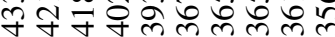
aicicicicicic

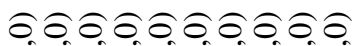

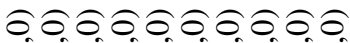

त० - in:00-1

min-miर mo

on in mit $=0$ min

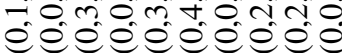

ésésésésés

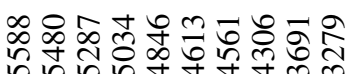

นำ

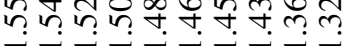

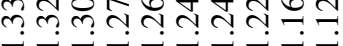

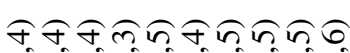
0.000
$00-00000=000$
000000 $0.00-00000$

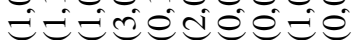

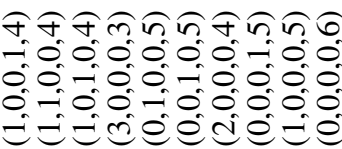

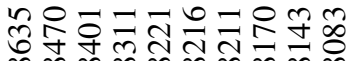
i i i i i i i i i i i

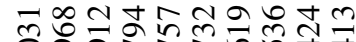

ลิอิดิดิอิ on-u-nmoजिंminmo -0.0 mi0.0.

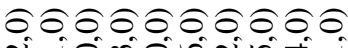
in $=0$ in 0 int(1)

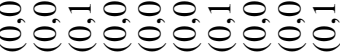

$\mathrm{N}$

$m$

n

$\simeq$

$\wedge$ $n$

$\infty$

m

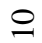




\subsection{Normal distribution}

Suppose that the underlying distribution is $N\left(0, \sigma^{2}\right)$. It can be easily shown that the MLE of $\sigma^{2}$ based on the SRS method, denoted by $\hat{\sigma}_{S R S}^{2}$, has the variance $\frac{2 \sigma^{4}}{n}$. On the other hand, as mentioned in subsection 3.1, the MLE of $\sigma^{2}$ on the basis of the FRSS scheme, i.e. $\hat{\sigma}_{F R S S}^{2}$, dose not have an explicit form. So, numerical simulations are required to study the behaviour of MSE. The simulation algorithm is performed with $2 \times 10^{5}$ repetitions for standard normal distribution. Moreover, to determine the values of $\operatorname{RE}\left(\hat{\sigma}_{F R S S}^{2}, \hat{\sigma}_{S R S}^{2}\right)$, some different costs have been considered. Values of $\operatorname{RE}\left(\hat{\sigma}_{F R S S}^{2}, \hat{\sigma}_{S R S}^{2}\right)$ are presented in Table 3 for some selected permutations of $\mathbf{m}$.

From Table 3, it is observed that:

- The estimator $\hat{\sigma}_{F R S S}^{2}$ is more efficient than $\hat{\sigma}_{S R S}^{2}$ for many permutations of $\mathbf{m}$.

- The optimal scheme is a combination of ERSS, MERSS type I and MERSS type II schemes. More precisely, when $k=4$ the optimal schemes $(0,1,5,0,0),(0,1,3,2,0)$ and $(0,4,2,0,0)$, are common for different values of costs in Table 3 and when $k=6$ the optimal common schemes are $(0,1,3,2,0)$ and $(0,0,6,0,0)$.

- When $k=6$, the RE of ERSS, $\mathbf{m}=(0,6,0,0,0)$, with respect to MERSS type $\mathbf{I}, \mathbf{m}=(0,0,6,0,0)$, in the problem of estimating $\sigma^{2}$ in normal distribution is $4.5179 / 4.4431=1.0168$. The REs of other schemes may be derived, similarly.

- The RE is sensitive to changes of the cost values and permutations order of $\mathbf{m}=\left(m_{1}, m_{2}, m_{3}, m_{4}, m_{5}\right)$ is changed by different values of costs.

\section{Conclusions}

In this paper, a FRSS scheme including ordinary RSS, ERSS, MERSS and MRSS was introduced and the associated likelihood function derived. The MLE was considered in scale family of distributions based on the FRSS. The existence and uniqueness of the MLEs of scale parameters were investigated in the exponential and normal distributions. Moreover, the amount of FI about the parameter of interest was studied and some results were presented in detail for the case of exponential distribution.

A comparison was done among different FRSS schemes. To obtain the optimal FRSS scheme a criterion was defined based on both efficiency and cost considerations. It was deduced that combining some existing sampling schemes increases the RE. Moreover, the optimal scheme depends on the underlying distribution and the parameter of interest. The proposed scheme can be extended to some other cases:

- Other versions of FRSS including more sampling schemes may be valuable to study.

- As previously mentioned in the procedure of ERSS, from one half of sets the minima and from the others the maxima are recorded. If in all sets the maxima are observed, using some algebraic and numerical computations, we observe that the amount of FI about the mean of exponential distribution increases.

\section{REFERENCES}

[1] W. Abu-Dayyeh and E. Al Sawi. Modified inference about the mean of the exponential distribution using moving extreme ranked set sampling. Statistical Papers, 50(2):249-259, 2009.

[2] M. Al-Odat and M. F. Al-Saleh. A variation of ranked set sampling. Journal of Applied Statistical Science, 10(2):137-146, 2001.

[3] A. I. Al-Omari and S. A. Al-Hadhrami. On maximum likelihood estimators of the parameters of a modified Weibull distribution using extreme ranked set sampling. Journal of Modern Applied Statistical Methods, 10(2):607-617, 2011.

[4] A. I. Al-Omari and M. Z. Raqab. Estimation of the population mean and median using truncation-based ranked set samples. Journal of Statistical Computation and Simulation, 83(8):1453-1471, 2013. 
[5] B. C. Arnold, N. Balakrishnan, and H. N. Nagaraja. A First Course in Order Statistics. SIAM, New York, 2008.

[6] S. Balci, A. D. Akkaya, and B. E. Ulgen. Modified maximum likelihood estimators using ranked set sampling. Journal of Computational and Applied Mathematics, 238:171-179, 2013.

[7] L. Barabesi and A. El-Sharaawi. The efficiency of ranked set sampling for parameter estimation. Statistics \& Probability Letters, 53(2):189-199, 2001.

[8] M. Chacko and P. Y. Thomas. Estimation of a parameter of bivariate Pareto distribution by ranked set sampling. Journal of Applied Statistics, 34(6):703-714, 2007.

[9] W. Chen, M. Xie, and M. Wu. Parametric estimation for the scale parameter for scale distributions using moving extremes ranked set sampling. Statistics \& Probability Letters, 83(9):2060-2066, 2013.

[10] W. Chen, M. Xie, and M. Wu. Modified maximum likelihood estimator of scale parameter using moving extremes ranked set sampling. Communications in Statistics-Simulation and Computation, 45(6):2232-2240, 2016.

[11] Z. Chen, Z. Bai, and B. Sinha. Ranked Set Sampling: Theory and Applications. Springer Science \& Business Media, 2003.

[12] T. Dell and J. Clutter. Ranked set sampling theory with order statistics background. Biometrics, 28(2):545$555,1972$.

[13] A. Eftekharian and M. Razmkhah. A unified ranked set sampling for estimating the population mean. ISTATISTIK: Journal of the Turkish Statistical Association, 9(3):107-118, 2016.

[14] G. McIntyre. A method for unbiased selective sampling, using ranked sets. Crop and Pasture Science, 3(4):385-390, 1952.

[15] R. Modarres and G. Zheng. Maximum likelihood estimation of dependence parameter using ranked set sampling. Statistics \& Probability Letters, 68(3):315-323, 2004.

[16] H. Muttlak. Median ranked set sampling. Journal of Applied Statistical Sciences, 6(4):245-255, 1997.

[17] H. Muttlak, S. Ahmed, and M. Al-Momani. Shrinkage estimation in replicated median ranked set sampling. Journal of Statistical Computation and Simulation, 80(11):1185-1196, 2010.

[18] R. W. Nahhas, D. A. Wolfe, and H. Chen. Ranked set sampling: cost and optimal set size. Biometrics, 58(4):964-971, 2002.

[19] H. M. Samawi, M. S. Ahmed, and W. Abu-Dayyeh. Estimating the population mean using extreme ranked set sampling. Biometrical Journal, 38(5):577-586, 1996.

[20] S. Sarikavanij, S. Kasala, B. K. Sinha, and M. Tiensuwan. Estimation of location and scale parameters in two-parameter exponential distribution based on ranked set sample. Communications in Statistics-Simulation and Computation, 43(1):132-141, 2014.

[21] A.-B. Shaibu and H. A. Muttlak. A comparison of the maximum likelihood estimators under ranked set sapling some of its modifications. Applied Mathematics and Computation, 129(2):441-453, 2002.

[22] L. Stokes. Parametric ranked set sampling. Annals of the Institute of Statistical Mathematics, 47(3):465-482, 1995.

[23] K. Takahasi and K. Wakimoto. On unbiased estimates of the population mean based on the sample stratified by means of ordering. Annals of the Institute of Statistical Mathematics, 20(1):1-31, 1968.

[24] Y.-G. Wang, Z. Chen, and J. Liu. General ranked set sampling with cost considerations. Biometrics, 60(2):556-561, 2004. 